\title{
A class of superconformal surfaces
}

\author{
M. Dajczer and Th. Vlachos
}

\begin{abstract}
Superconformal surfaces in Euclidean space are the ones for which the ellipse of curvature at any point is a nondegenerate circle. They can be characterized as the surfaces for which a well-known pointwise inequality relating the intrinsic Gauss curvature with the extrinsic normal and mean curvatures, due to Wintgen ([22]) and Guadalupe-Rodríguez ([13]) for any codimension, reaches equality at all points. In this paper, we show that any pedal surface to a 2 -isotropic Euclidean surface is superconformal. Opposed to almost all known examples, superconformal surfaces in this class are not conformally equivalent to minimal surfaces. Moreover, they can be given in an explicit parametric form since 2 -isotropic surfaces admit a Weierstrass type representation.
\end{abstract}

A surface in Euclidean space $f: M^{2} \rightarrow \mathbb{R}^{n}, n \geq 4$, is called superconformal if at any point the ellipse of curvature is a nondegenerate circle. The ellipse of curvature $\mathcal{E}(p)$ of $f$ at $p \in M^{2}$ is the ellipse in the normal space $N_{f} M(p)$ given as the image of the second fundamental form $\alpha_{f}$ restricted to the unit circle in the tangent plane $T_{p} M$, i.e.,

$$
\mathcal{E}(p)=\left\{\alpha_{f}(X, X): X \in T_{p} M,\|X\|=1\right\} ;
$$

see [13] and references therein for several facts on this concept whose study started almost a century ago due to the work of Moore and Wilson [16], [17. Superconformality is invariant under conformal (Moebius) transformations since the property of $\mathcal{E}(p)$ being a circle is invariant under conformal changes of the metric of the ambient space.

Perhaps the most notable property of superconformality is that the surfaces yield equality in the pointwise inequality due to Wintgen [22] for $n=4$ and GuadalupeRodríguez [13] for any codimension, that reads as follows:

$$
K+\left|K_{N}\right| \leq\|H\|^{2}
$$

where $K$ denotes the Gaussian curvature of $M^{2}$ and $K_{N}$ and $H$ are the normal curvature and mean curvature vector of $f$, respectively.

In the special case of surfaces $f: M^{2} \rightarrow \mathbb{R}^{4}$ the condition of superconformality is rather strong. This allowed Dajczer and Tojeiro [9] to provide a parametric representation of all non-minimal superconformal surface in terms of any minimal surface and 
its conjugate. Notice that minimal superconformal surfaces in $\mathbb{R}^{4}$ are just holomorphic curves with respect to some complex structure in the ambient space.

In higher codimension superconformality is not longer such a strong assumption and several classes of examples of such surfaces have been considered. The "trivial" examples are the ones conformally equivalent (i.e., congruent by a Moebius transformation) to minimal superconformal surfaces. These Euclidean surfaces are called 1-isotropic and admit a Weierstrass type representation as, in fact, does any simply connected $s$-isotropic surface; see next section for definitions and some properties including a parametric representation of these minimal surfaces.

Other examples of superconformal surfaces are the images by stereographic projection of minimal superconformal surfaces in the sphere and hyperbolic space. In the spherical case, this class of surfaces has been studied in different contexts; see [2], [15] and [21. In particular, it includes the minimal 2-spheres studied in [1], [4] and [6], the holomorphic curves in the nearly Kaehler sphere $\mathbb{S}^{6}[3]$ and Lawson's surfaces [21] for appropriate choice of the several parameters involved in their definition. There are also the super Willmore surfaces in even dimensional spheres introduced as isotropic S-Willmore surfaces by Ejiri [11] and classified in terms of isotropic holomorphic curves in complex projective spaces. It was shown recently [10] that superconformal S-Willmore surfaces are the ones to have a dual surface in the same class. Finally, the case of the hyperbolic ambient space was studied in [14.

All of the above "nontrivial" examples have in common that they do not posses an explicit parametric description. Moreover, with the exception of the super Willmore surfaces, they are all conformally equivalent to minimal surfaces. In this paper, we present a new class of superconformal surfaces which do admit an explicitly parametric representation and are never locally conformally equivalent to minimal surfaces.

The pedal surface $g: M^{2} \rightarrow \mathbb{R}^{n}$ to a surface $f: M^{2} \rightarrow \mathbb{R}^{n}$ is defined as the locus of the foot of the perpendicular from a fixed arbitrary point to the tangent planes to $f$ and assumed to be an immersion. For surfaces in $\mathbb{R}^{3}$ this concept was considered by several authors during the second half of XIX century; see [12, p. 673] and [19, pp. 155-161].

Theorem 1. A pedal surface $g: M^{2} \rightarrow \mathbb{R}^{n}, n \geq 6$, to a substantial 1-isotropic surface $f: M^{2} \rightarrow \mathbb{R}^{n}$ is superconformal if and only $f$ is 2-isotropic. Then, we have:

(i) The surfaces $f$ and $g$ are conformal.

(ii) The surface $g$ is not locally conformally equivalent to a minimal surface.

(iii) The surface $g$ is never $S$-Willmore.

In particular, the above result applies to any holomorphic curve $f: M^{2} \rightarrow \mathbb{C}^{m}$ for $m \geq 3$, since these surfaces are the ones that are $(m-1)$-isotropic in $\mathbb{R}^{2 m}$. By $f$ being substantial we mean that the surface is not contained in any proper affine subspace. 
It was shown by Rouxel [18] that a pedal surface to any holomorphic curve in $\mathbb{C}^{2} \cong \mathbb{R}^{4}$ is a superconformal surface. But opposed to part $(i i)$ in the theorem he also proved that the pedal surface is conformally equivalent to an holomorphic curve with respect to some conformal structure in $\mathbb{R}^{4}$. Besides, the proof of these two facts can be easily obtained from the arguments in this paper.

\section{Preliminaries}

Let $f: M^{2} \rightarrow \mathbb{R}^{n}, n \geq 4$, be an isometric immersion of a two-dimensional Riemannian manifold into $n$-dimensional Euclidean space. The $k^{\text {th }}$-normal space of the surface $f$ at $p \in M^{2}$ for $k \geq 1$ is defined as

$$
N_{k}^{f}(p)=\operatorname{span}\left\{\alpha_{f}^{k+1}\left(X_{1}, \ldots, X_{k+1}\right): X_{1}, \ldots, X_{k+1} \in T_{p} M\right\}
$$

where $\alpha_{f}^{2}=\alpha_{f}: T M \times T M \rightarrow N_{f} M$ is the standard second fundamental form and

$$
\alpha_{f}^{s}: T M \times \cdots \times T M \rightarrow N_{f} M, s \geq 3
$$

denotes the symmetric tensor called the $s^{\text {th }}$-fundamental form defined inductively by

$$
\alpha_{f}^{s}\left(X_{1}, \ldots, X_{s}\right)=\left(\nabla_{X_{s}}^{\perp} \ldots \nabla_{X_{3}}^{\perp} \alpha_{f}\left(X_{2}, X_{1}\right)\right)^{\perp}
$$

where ()$^{\perp}$ means taking the projection onto the normal subspace $\left(N_{1}^{f} \oplus \ldots \oplus N_{s-2}^{f}\right)^{\perp}$.

Since this paper deals with local theory, we always assume that the immersion $f$ is regular (called nicely curved in [20]) which means that all $N_{k}^{f}$ 's have constant dimension for each $k$ and thus form normal subbundles. Notice that this condition is verified along connected components of an open dense subset of $M^{2}$.

Assume now that the surface $f$ is minimal. In this case, the normal bundle splits as

$$
N_{f} M=N_{1}^{f} \oplus N_{2}^{f} \oplus \cdots \oplus N_{m}^{f}, \quad m=[(n-1) / 2],
$$

since all higher normal bundles have rank two except the last one that has rank one if $n$ is odd; see [6], [7] or [20]. Moreover, the orientation of $M^{2}$ and $\alpha_{f}^{s}$ induce an orientation on each plane bundle $N_{s-1}^{f}$ given by the ordered pair

$$
\left\{\alpha_{f}^{s}(X, \ldots, X), \alpha_{f}^{s}(J X, \ldots, X)\right\}
$$

where $J$ is the complex structure of $M^{2}$ determined by the metric and the orientation.

Each two dimensional normal subbundle in (1) has associate an ellipse of curvature defined as follows: The $s^{t h}$-order ellipse of curvature $\mathcal{E}_{s}^{f}(p) \subset N_{s}^{f}(p), 1 \leq s \leq m_{0}$, of $f$ at $p \in M^{2}$ is

$$
\mathcal{E}_{s}^{f}(p)=\left\{\alpha_{f}^{s+1}\left(X_{\psi}, \ldots, X_{\psi}\right): X_{\psi}=\cos \psi X+\sin \psi J X \text { and } \psi \in[0,2 \pi)\right\}
$$


where $X \in T_{p} M$ has unit length and $N_{m_{0}}^{f}$ is the last plane subbundle. Of course, the standard ellipse of curvature is just $\mathcal{E}(p)=\mathcal{E}_{1}^{f}(p)$.

The minimal surface $f$ is called $m$-isotropic if $\mathcal{E}_{r}^{f}(p)$ is a circle for any $p \in M^{2}$ and any $1 \leq r \leq m$. Thus minimal superconformal surfaces are the 1-isotropic ones. A fundamental fact in relation to this paper is that any simply connected $m$-isotropic surface admit a Weierstrass type representation given in [8] based on results in [5].

Start with a nonzero holomorphic map

$$
\alpha_{0}: U \rightarrow \mathbb{C}^{N-2(m+1)}
$$

Assuming that

$$
\alpha_{r}: U \rightarrow \mathbb{C}^{N-2(m-r+1)}, \quad 0 \leq r \leq m,
$$

has been defined already, set

$$
\alpha_{r+1}=\beta_{r+1}\left(1-\phi_{r}^{2}, i\left(1+\phi_{r}^{2}\right), 2 \phi_{r}\right)
$$

where $\phi_{r}=\int^{z} \alpha_{r} d z$ and $\beta_{r+1} \neq 0$ is any holomorphic function. Then, the surface $g=\operatorname{Re} \phi_{m+1}$ in $\mathbb{R}^{N}$ is $m$-isotropic.

\section{The proof}

Given a surface $f: M^{2} \rightarrow \mathbb{R}^{n}, n \geq 4$, set $\alpha_{i j}=\alpha_{f}\left(X_{i}, X_{j}\right)$ where $\left\{X_{1}, X_{2}\right\}$ is an orthonormal basis of $T_{p} M$ at $p \in M^{2}$. For a unit vector $v=\cos \theta X_{1}+\sin \theta X_{2}$, we have

$$
\alpha_{f}(v, v)=H+\cos 2 \theta \xi_{1}+\sin 2 \theta \xi_{2}
$$

where $\xi_{1}=\frac{1}{2}\left(\alpha_{11}-\alpha_{22}\right), \xi_{2}=\alpha_{12}$ and $H$ is the mean curvature vector. Thus $\mathcal{E}(p)$ is a circle if and only if we have

$$
\left\langle\alpha_{12}, \alpha_{11}-\alpha_{22}\right\rangle=0 \text { and }\left\|\alpha_{11}-\alpha_{22}\right\|=2\left\|\alpha_{12}\right\| .
$$

The complexified tangent bundle $T M \otimes \mathbb{C}$ decomposes into the eigenspaces of the complex structure $J$ corresponding to the eigenvalues $i$ and $-i$ denoted by $T^{\prime} M$ and $T^{\prime \prime} M$, respectively. The second fundamental form can be complex linearly extended to $T M \otimes \mathbb{C}$ with values in the complexified vector bundle $N_{f} M \otimes \mathbb{C}$ and then decomposed into its $(p, q)$-components, $p+q=2$, which are tensor products of $p$ many 1 -forms vanishing on $T^{\prime \prime} M$ and $q$ many 1 -forms vanishing on $T^{\prime} M$.

Taking local isothermal coordinates $\{x, y\}$ and $\partial=(1 / 2)\left(\partial_{x}-i \partial_{y}\right)$, we have from (2) that $f$ is superconformal if and only if the $(2,0)$-part of the second fundamental form is isotropic, i.e., the complex line bundle spanned by $\alpha_{f}(\partial, \partial)$ is isotropic. Moreover, the surface is minimal if its $(1,1)$-part of $\alpha_{f}$ vanishes, i.e., if $\alpha_{f}(\partial, \bar{\partial})=0$. 
Without loss of generality, we choose to take the pedal surface always with respect to the origin since changing that amounts to add a constant vector to $f$; see last section for further discussion on this election.

Given a substantial minimal surface $f: M^{2} \rightarrow \mathbb{R}^{n}, n \geq 6$, decompose the position vector into its tangent and normal components

$$
f=f_{*} Z+g
$$

and assume that $g: M^{2} \rightarrow \mathbb{R}^{n}$ is an immersion. Thus $g$ is the pedal surface of $f$ with respect to the origin. Differentiating (3) yields

$$
X=\nabla_{X} Z-A_{g} X \text { and } \alpha(X, Z)+\nabla_{X}^{\perp} g=0
$$

where $A_{g}=A_{g}^{f}$ is the shape operator associated to $g$ and $\alpha=\alpha_{f}$. Moreover, we had identified $X$ with $f_{*} X$ for $X \in T M$. It follows that

$$
g_{*} X=-A_{g} X-\alpha(X, Z) .
$$

Hereafter, we assume that $Z \neq 0$ and $g \notin\left(N_{1}^{f}\right)^{\perp}$ at any point. Clearly, we cannot have $Z=0$ in an open subset. On an open subset where $g \in\left(N_{1}^{f}\right)^{\perp}$ it is easy to see from the first equation in (44) that $M^{2}$ would be flat and $f$ totally geodesic. We denote $g=\delta+\eta$ where $\delta \neq 0$ and $\eta$ are the $N_{1}^{f}$-component and $\left(N_{1}^{f}\right)^{\perp}$-component of $g$, respectively.

Lemma 2. A minimal surface $f: M^{2} \rightarrow \mathbb{R}^{n}$ is conformal to its pedal surface if and only if it is 1-isotropic. In this case, we have $d s_{g}^{2}=-(1 / 2) K \theta d s_{f}^{2}$ where $K$ is the Gaussian curvature of $M^{2}$ and $\theta=\|Z\|^{2}+\|\delta\|^{2}$.

Proof: In general, we have that $g$ is conformal to $f$ if and only if $\left\langle g_{*} \partial, g_{*} \partial\right\rangle=0$. Since $f$ is minimal, we obtain from (5) that

$$
\left\langle g_{*} X, g_{*} Y\right\rangle=-\operatorname{det} A_{\delta}\langle X, Y\rangle+\langle\alpha(X, Z), \alpha(Y, Z)\rangle
$$

for any $X, Y \in T M$. Thus $g$ is conformal to $f$ if and only if $\langle\alpha(\partial, Z), \alpha(\partial, Z)\rangle=0$. Being $f$ minimal, we have $i \alpha(\partial, Z)=\alpha(\partial, J Z)$. Hence $g$ is conformal to $f$ if and only if

$$
\langle\alpha(\partial, Z-i J Z), \alpha(\partial, Z-i J Z)\rangle=0,
$$

and this is equivalent to $f$ being 1-isotropic since $Z-i J Z \in T^{\prime} M=\operatorname{span}_{\mathbb{C}}\{\partial\}$.

We compute the conformal factor. We have

$$
\langle\partial, \bar{\partial}\rangle_{g}=-\operatorname{det} A_{\delta}\langle\partial, \bar{\partial}\rangle+\langle\alpha(\partial, Z), \alpha(\bar{\partial}, Z)\rangle
$$

From the Gauss equation and since $f$ is minimal, it follows that

$$
\langle\alpha(\partial, Z), \alpha(\bar{\partial}, Z)\rangle=-\langle R(\partial, Z) Z, \bar{\partial}\rangle=K\left(|\langle\partial, Z\rangle|^{2}-\|Z\|^{2}\langle\partial, \bar{\partial}\rangle\right)=-\frac{1}{2} K\|Z\|^{2}\langle\partial, \bar{\partial}\rangle .
$$


where $R$ is the curvature tensor of $M^{2}$. Since $f$ is 1-isotropic, given an orthonormal $\left\{e_{3}, e_{4}\right\}$ frame in $N_{1}^{f}$ there is an orthonormal tangent frame such that

$$
A_{e_{3}}=\left(\begin{array}{cc}
\kappa & 0 \\
0 & -\kappa
\end{array}\right), \quad A_{e_{4}}=\left(\begin{array}{cc}
0 & \kappa \\
\kappa & 0
\end{array}\right) .
$$

Thus $\operatorname{det} A_{\delta}=(1 / 2) K\|\delta\|^{2}$, and the claim follows.

Lemma 3. Assume that $f$ is 1-isotropic. Then, the normal bundle of $g$ is given by

$$
N_{g} M=\operatorname{span}\left\{Z-\delta, J Z+J_{1}^{\perp} \delta\right\} \oplus\left(N_{1}^{f}\right)^{\perp}
$$

where $J_{1}^{\perp}$ denotes the orthogonal complex structure of $N_{1}^{f}$.

Proof: That $f$ is 1-isotropic is equivalent to

$$
J_{1}^{\perp} \alpha(X, Y)=\alpha(J X, Y)
$$

for any $X, Y \in T M$. Then

$$
\left\langle g_{*} X, J Z+J_{1}^{\perp} \delta\right\rangle=\left\langle J A_{\delta} X+J_{1}^{\perp} \alpha(X, Z), Z+\delta\right\rangle=-\left\langle A_{\delta} J X, Z\right\rangle+\langle\alpha(J X, Z), \delta\rangle=0
$$

and the remaining cases are immediate.

We define a complex structure $\mathcal{J}=J_{1}^{\perp} \oplus J_{2}^{\perp}$ on $N_{1}^{f} \oplus N_{2}^{f}$ where $J_{2}^{\perp}$ denotes the orthogonal complex structure of $N_{2}^{f}$.

Lemma 4. If $f$ is 1-isotropic, then the following equations hold:

$$
\left(\nabla_{X}^{\perp} \mathcal{J}\right) e_{3}=(\lambda-1)\left(* \omega(X) e_{5}+\omega(X) e_{6}\right), \quad\left(\nabla_{X}^{\perp} \mathcal{J}\right) e_{4}=(\lambda-1)\left(\omega(X) e_{5}-* \omega(X) e_{6}\right)
$$

where $0<\lambda \leq 1$ is the ratio of the length of the axes of $\mathcal{E}_{2}^{f}$. Moreover, the orthonormal frames $\left\{e_{3}, e_{4}\right\}$ and $\left\{e_{5}, e_{6}\right\}$ span $N_{1}^{f}$ and $N_{2}^{f}$, respectively, $\omega=\left\langle\nabla^{\perp} e_{3}, e_{5}\right\rangle$ and $*$ is the Hodge operator.

Proof: Arguing as in the proof of Lemma 5 in [21] we obtain that there is a local orthonormal frame $\left\{e_{A}\right\}, 1 \leq A \leq n$, where $\left\{e_{1}, e_{2}\right\}$ is a tangent frame and $\left\{e_{2 r+1}, e_{2 r+2}\right\}$ is a positively oriented frame of $N_{r}^{f}$ for $1 \leq r \leq m_{0}$, such that the normal connection forms $\omega_{\alpha \beta}=\left\langle\nabla^{\perp} e_{\alpha}, e_{\beta}\right\rangle$ satisfy

$$
\omega_{45}=-* \omega, \quad \omega_{46}=-* \omega_{36}, \quad \omega_{36}=\lambda * \omega \text { and } \omega_{46}=\lambda * \omega_{45} .
$$

Moreover, we have that $\left(\nabla_{X}^{\perp} \mathcal{J}\right) e_{\alpha} \in N_{2}^{f}$ for $3 \leq \alpha \leq 4$, and the remaining of the proof is straightforward. 
Lemma 5. If $f$ is 1-isotropic, then the second fundamental form of $g$ satisfies

$$
\alpha_{g}(\partial, \partial)=\left\langle\alpha_{g}(\partial, \partial), \xi_{1}\right\rangle\left(\xi_{1}+i \xi_{2}\right)-\omega(\partial)\left\langle\alpha(\partial, Z), e_{3}+i e_{4}\right\rangle\left(e_{5}-i \lambda e_{6}\right)
$$

where $\xi_{1}=(Z-\delta) / \sqrt{\theta}$ and $\xi_{2}=(J Z+\mathcal{J} \delta) / \sqrt{\theta}$.

Proof: From

$$
\tilde{\nabla}_{X} g_{*} Y=-\nabla_{X} A_{\delta} Y+A_{\alpha(Y, Z)} X-\alpha\left(X, A_{\delta} Y\right)-\nabla_{X}^{\perp} \alpha(Y, Z)
$$

we obtain that

$$
\left(\alpha_{g}(X, Y)\right)^{\left(N_{1}^{f}\right)^{\perp}}=-\left(\nabla_{X}^{\perp} \alpha(Y, Z)\right)^{\left(N_{1}^{f}\right)^{\perp}} .
$$

Hence, $\left\langle\alpha_{g}(\partial, \partial), \nu\right\rangle=-\left\langle\nabla_{\partial}^{\perp} \alpha(\partial, Z), \nu\right\rangle$ for any $\nu \in\left(N_{1}^{f}\right)^{\perp}$. In particular,

$$
\left\langle\alpha_{g}(\partial, \partial), \nu\right\rangle=0 \text { for any } \nu \in\left(N_{1}^{f} \oplus N_{2}^{f}\right)^{\perp} \text {. }
$$

On the other hand, since $\alpha\left(\partial, A_{\delta} \partial\right)=0$, we have

$$
\left\langle\alpha_{g}(\partial, \partial), Z-\delta\right\rangle=\left\langle-\nabla_{\partial} A_{\delta} \partial+A_{\alpha(\partial, Z)} \partial, Z\right\rangle+\left\langle\nabla_{\partial}^{\perp} \alpha(\partial, Z), \delta\right\rangle
$$

and, using Lemma 4, that

$$
\begin{aligned}
\left.\left\langle\alpha_{g}(\partial, \partial), J Z+\mathcal{J} \delta\right)\right\rangle & =-\left\langle\nabla_{\partial} A_{\delta} \partial-A_{\alpha(\partial, Z)} \partial, J Z\right\rangle-\left\langle\nabla_{\partial}^{\perp} \alpha(\partial, Z), \mathcal{J} \delta\right\rangle \\
& =\left\langle J \nabla_{\partial} A_{\delta} \partial, Z\right\rangle+\left\langle A_{\alpha(\partial, Z)} J \partial, Z\right\rangle+\left\langle\mathcal{J} \nabla_{\partial}^{\perp} \alpha(\partial, Z), \delta\right\rangle \\
& =-\left\langle\nabla_{\partial} A_{\delta} J \partial, Z\right\rangle+i\left\langle A_{\alpha(\partial, Z)} \partial, Z\right\rangle+\left\langle\nabla_{\partial}^{\perp} \mathcal{J} \alpha(\partial, Z), \delta\right\rangle \\
& =-i\left\langle\nabla_{\partial} A_{\delta} \partial, Z\right\rangle+i\left\langle A_{\alpha(\partial, Z)} \partial, Z\right\rangle+i\left\langle\nabla_{\partial}^{\perp} \alpha(\partial, Z), \delta\right\rangle \\
& =i\left\langle\alpha_{g}(\partial, \partial), Z-\delta\right\rangle
\end{aligned}
$$

It follows that

$$
\alpha_{g}(\partial, \partial)=\left\langle a_{g}(\partial, \partial), \xi_{1}\right\rangle\left(\xi_{1}+i \xi_{2}\right)+\left\langle\alpha_{g}(\partial, \partial), e_{5}\right\rangle e_{5}+\left\langle\alpha_{g}(\partial, \partial), e_{6}\right\rangle e_{6}
$$

Moreover, we obtain using (7) that

$$
\begin{aligned}
\left\langle\alpha_{g}(\partial, \partial), e_{5}\right\rangle=\left\langle\alpha(\partial, Z), \nabla_{\partial}^{\perp} e_{5}\right\rangle & =-\left\langle\alpha(\partial, Z), \omega(\partial) e_{3}-* \omega(\partial) e_{4}\right\rangle \\
& =-\omega(\partial)\left\langle\alpha(\partial, Z), e_{3}+i e_{4}\right\rangle .
\end{aligned}
$$

Similarly,

$$
\left\langle\alpha_{g}(\partial, \partial), e_{6}\right\rangle=i \lambda \omega(\partial)\left\langle\alpha(\partial, Z) e_{3}+i e_{4}\right\rangle,
$$

and the result follows.

Proof of Theorem 1: By Lemma 5 the pedal surface is superconformal if and only if

$$
\left(1-\lambda^{2}\right)\left(\omega(\partial)\left\langle\alpha(\partial, Z), e_{3}+i e_{4}\right\rangle\right)^{2}=0
$$


But if $\left\langle\alpha(\partial, Z), e_{3}+i e_{4}\right\rangle=0$, then $Z=0$ and that is a contradiction. And if $\omega(\partial)=0$ we have from (7) that $N_{1}^{f}$ is parallel in the normal bundle, also a contradiction. We conclude that $\lambda=1$.

From Lemma 2, the mean curvature vector field of $g$ is

$$
H_{g}=-\frac{1}{K \theta} \Delta g
$$

where the Laplace operator of $M^{2}$ acts on vector valued functions. Using (5), we have

$$
\Delta g=-\sum_{j=1,2}\left(\left(\nabla_{e_{j}} A_{\delta}\right) e_{j}-A_{\alpha\left(e_{j}, Z\right)} e_{j}+\alpha\left(e_{j}, A_{\delta} e_{j}\right)+\nabla_{e_{j}}^{\perp} \alpha\left(e_{j}, Z\right)-\alpha\left(\nabla_{e_{j}} e_{j}, Z\right)\right)
$$

for an orthonormal frame $\left\{e_{1}, e_{2}\right\}$ in $T M$. Using the Codazzi equation, we have

$$
\begin{aligned}
& \sum_{j=1,2}\left\langle\left(\nabla_{e_{j}} A_{\delta}\right) e_{j}, X\right\rangle=\sum_{j=1,2}\left\langle\left(\nabla_{e_{j}} A_{\delta}\right) X, e_{j}\right\rangle \\
&=\sum_{j=1,2}\left\langle\left(\nabla_{X} A_{\delta}\right) e_{j}, e_{j}\right\rangle+\sum_{j=1,2}\left\langle A_{\nabla_{e_{j}} \delta} X, e_{j}\right\rangle-\sum_{j=1,2}\left\langle A_{\nabla_{X}} \delta\right. \\
&\left.e_{j}, e_{j}\right\rangle \\
&=\sum_{j=1,2}\left\langle\left(\nabla_{X} A_{\delta}\right) e_{j}, e_{j}\right\rangle-\sum_{j=1,2}\left\langle A_{\alpha\left(e_{j}, Z\right)} X, e_{j}\right\rangle .
\end{aligned}
$$

On the other hand,

$$
\begin{aligned}
\sum_{j=1,2}\left\langle\left(\nabla_{X} A_{\delta}\right) e_{j}, e_{j}\right\rangle & =\sum_{j=1,2}\left\langle\nabla_{X} A_{\delta} e_{j}, e_{j}\right\rangle-\sum_{j=1,2}\left\langle A_{\delta} \nabla_{X} e_{j}, e_{j}\right\rangle \\
& =\sum_{j=1,2} X\left\langle A_{\delta} e_{j}, e_{j}\right\rangle-2 \sum_{j=1,2}\left\langle A_{\delta} \nabla_{X} e_{j}, e_{j}\right\rangle \\
& =0 .
\end{aligned}
$$

We obtain that

$$
\Delta g=\sum_{j=1,2}\left(2 A_{\alpha\left(e_{j}, Z\right)} e_{j}-\alpha\left(e_{j}, A_{\delta} e_{j}\right)-\nabla_{e_{j}}^{\perp} \alpha\left(e_{j}, Z\right)+\alpha\left(\nabla_{e_{j}} e_{j}, Z\right)\right) .
$$

Choosing orthonormal frames $\left\{e_{3}, e_{4}\right\}$ in $N_{1}^{f}$ with $e_{3}=\delta /\|\delta\|$ and $\left\{e_{1}, e_{2}\right\}$ in $T M$ such that (6) holds, we have

$$
\sum_{j=1,2} A_{\alpha\left(e_{j}, Z\right)} e_{j}=-K Z \text { and } \sum_{j=1,2} \alpha\left(e_{j}, A_{\delta} e_{j}\right)=-K \delta .
$$

A straightforward computation using (44), (7) and the Codazzi equation for $A_{e_{3}}$, that is,

$$
e_{1}(\kappa)=-2 \kappa \psi\left(e_{2}\right)+\kappa \omega_{34}\left(e_{2}\right) \text { and } e_{2}(\kappa)=2 \kappa \psi\left(e_{1}\right)-\kappa \omega_{34}\left(e_{1}\right) \text {, }
$$


gives that

$$
\sum_{j=1,2} \nabla_{e_{j}}^{\perp} \alpha\left(e_{j}, Z\right)=-K \delta-\kappa\left(\left(z_{1} \psi\left(e_{2}\right)+z_{2} \psi\left(e_{1}\right)\right) e_{3}-\left(z_{1} \psi\left(e_{1}\right)-z_{2} \psi\left(e_{2}\right)\right) e_{4}\right)
$$

where $Z=z_{1} e_{1}+z_{2} e_{2}$ and $\psi=\left\langle\nabla e_{1}, e_{2}\right\rangle$. Moreover,

$$
\sum_{j=1,2} \alpha\left(\nabla_{e_{j}} e_{j}, Z\right)=-\kappa\left(\left(z_{1} \psi\left(e_{2}\right)+z_{2} \psi\left(e_{1}\right)\right) e_{3}-\left(z_{1} \psi\left(e_{1}\right)-z_{2} \psi\left(e_{2}\right)\right) e_{4}\right) .
$$

Now, we obtain from (11) that $\Delta g=2 K(\delta-Z)$ and, consequently, that

$$
H_{g}=\frac{2}{\theta}(Z-\delta)
$$

Consider the inversion $\mathcal{I}$ with respect to a sphere with radius $R$ centered at $p_{0}$ and the immersion $\tilde{g}=\mathcal{I} \circ g$. Then, there is a vector bundle isometry $\mathcal{P}$ between the normal bundles $N_{g} M$ and $N_{\tilde{g}} M$ (see [9]) given by

$$
\mathcal{P} \mu=\mu-2 \frac{\left\langle g-p_{0}, \mu\right\rangle}{\left\|g-p_{0}\right\|^{2}}\left(g-p_{0}\right)
$$

such that shape operators of $g$ and $\tilde{g}$ are related by

$$
\tilde{A}_{\mathcal{P} \mu}=\frac{1}{R^{2}}\left(\left\|g-p_{0}\right\|^{2} A_{\mu}+2\left\langle g-p_{0}, \mu\right\rangle I\right) .
$$

Then, the mean curvature vector of $\tilde{g}$ is given by

$$
H_{\tilde{g}}=\frac{1}{R^{2}} \mathcal{P}\left(\left\|g-p_{0}\right\|^{2} H_{g}+2\left(g-p_{0}\right)^{\perp}\right) .
$$

Using Lemma 3, we have

$$
\begin{aligned}
\frac{1}{2}\left\|g-p_{0}\right\|^{2} H_{g}+\left(g-p_{0}\right)^{\perp}= & \frac{1}{\theta}\left(\left\|g-p_{0}\right\|^{2}-\|\delta\|^{2}-\left\langle p_{0}, Z-\delta\right\rangle\right)(Z-\delta) \\
& -\frac{1}{\theta}\left\langle p_{0}, J Z+\mathcal{J} \delta\right\rangle(J Z+\mathcal{J} \delta)+\eta-\left(p_{0}\right)^{\left(N_{1}^{f}\right)^{\perp}} .
\end{aligned}
$$

Thus $\tilde{g}$ is minimal if and only if

$$
\left\|g-p_{0}\right\|^{2}-\|\delta\|^{2}-\left\langle p_{0}, Z-\delta\right\rangle=0,\left\langle p_{0}, J Z+\mathcal{J} \delta\right\rangle=0 \text { and } \eta=\left(p_{0}\right)^{\left(N_{1}^{f}\right)^{\perp}} .
$$

If $p_{0}=0$, then $g \in N_{1}^{f}$ and (4) combined with Lemma 4 yield that $N_{1}^{f}=\operatorname{span}\{\delta, \mathcal{J} \delta\}$ is parallel in the normal bundle, which has been excluded. Thus, we may assume $p_{0} \neq 0$. Setting $p_{0}=W+\xi+\eta$ where $W \in T M$ and $\xi \in N_{1}^{f}$, we have

$$
\nabla_{X} W=A_{\xi} X \text { and } \alpha(X, W)=-\nabla_{X}^{\perp} \xi-\nabla_{X}^{\perp} \eta
$$


From (44) we obtain $\nabla_{X}^{\perp}(\delta-\xi) \in N_{1}^{f}$ and if $\delta \neq \xi$ the same argument as before gives that $N_{1}^{f}$ is parallel in the normal bundle. Hence $\xi=\delta$. Hence, the second equation in (13) gives that $W$ and $Z$ are collinear. But then the first equation in (13) yields $W=Z$, and this is not possible by (44) and (14). Thus $\tilde{g}$ cannot be minimal.

To conclude the proof we show that $g$ is never S-Willmore. By definition, we have that $g$ is S-Willmore if and only if $\hat{\nabla} \frac{\perp}{\bar{\partial}} \alpha_{g}(\partial, \partial)$ is parallel to $\alpha_{g}(\partial, \partial)$ where $\hat{\nabla}^{\perp}$ is the normal connection of $g$. The Codazzi equation gives

$$
\hat{\nabla} \frac{\perp}{\partial} H_{g}=\frac{2}{\rho^{2}} \hat{\nabla} \frac{\perp}{\bar{\partial}} \alpha_{g}(\partial, \partial)
$$

where the induced metric is $d s_{g}^{2}=\rho^{2}|d z|^{2}$ and $z=x+i y$. Therefore, the superconformal surface $g$ is S-Willmore if and only if $\hat{\nabla} \frac{\perp}{\partial} H_{g}$ is parallel to $\alpha_{g}(\partial, \partial)$.

By Lemma 5, we have

$$
\alpha_{g}(\partial, \partial)=\left\langle\alpha_{g}(\partial, \partial), \xi_{1}\right\rangle\left(\xi_{1}+i \xi_{2}\right)-\omega(\partial)\left\langle\alpha(\partial, Z), e_{3}+i e_{4}\right\rangle\left(e_{5}-i \lambda e_{6}\right) .
$$

We claim that

$$
\left\langle\alpha_{g}(\partial, \partial), \xi_{1}\right\rangle=\frac{1}{\sqrt{\theta}}\left(-\partial(\|\delta\|)\left\langle A_{e_{3}} \partial, Z\right\rangle+\|\delta\|\left\langle A_{e_{3}} \partial, \partial\right\rangle-\|\delta\| \omega_{34}(\partial)\left\langle A_{e_{4}} \partial, Z\right\rangle\right) .
$$

First observe that since $f$ is 1 -isotropic, we have

$$
\left\langle A_{\alpha(\partial, Z)} \partial, Z\right\rangle=\left\langle A_{e_{3}} \partial, Z\right\rangle^{2}+\left\langle A_{e_{4}} \partial, Z\right\rangle^{2}=0 .
$$

Now from (9) using (4), we obtain

$$
\begin{aligned}
\left\langle\alpha_{g}(\partial, \partial), \xi_{1}\right\rangle & =\frac{1}{\sqrt{\theta}}\left(-\left\langle\nabla_{\partial} A_{\delta} \partial, Z\right\rangle+\left\langle\nabla_{\partial}^{\perp} \alpha(\partial, Z), \delta\right\rangle\right) \\
& =\frac{1}{\sqrt{\theta}}\left(-\left\langle\nabla_{\partial} A_{\delta} \partial, Z\right\rangle+\partial\langle\alpha(\partial, Z), \delta\rangle-\left\langle\alpha(\partial, Z), \nabla_{\partial}^{\perp} \delta\right\rangle\right) \\
& =\frac{1}{\sqrt{\theta}}\left(-\left\langle\nabla_{\partial} A_{\delta} \partial, Z\right\rangle+\partial\left\langle A_{\delta} \partial, Z\right\rangle-\left\langle\alpha(\partial, Z), \nabla_{\partial}^{\perp}\left(\|\delta\| e_{3}\right)\right\rangle\right) \\
& =\frac{1}{\sqrt{\theta}}\left(\left\langle A_{\delta} \partial, \partial\right\rangle+\left\langle A_{\delta} \partial, A_{\delta} \partial\right\rangle-\partial(\|\delta\|)\left\langle A_{e_{3}} \partial, Z\right\rangle-\|\delta\| \omega_{34}(\partial)\left\langle A_{e_{4}} \partial, Z\right\rangle\right),
\end{aligned}
$$

and the claim follows.

On the other hand, the second equation in (4) using (7) yields

$$
\left\langle A_{e_{3}} \partial, Z\right\rangle=-\partial\|\delta\|+\omega(\partial)\left(\eta_{5}-i \eta_{6}\right) \text { and }\left\langle A_{e_{4}} \partial, Z\right\rangle=-\|\delta\| \omega_{34}(\partial)+i \omega(\partial)\left(\eta_{5}-i \eta_{6}\right)
$$

where $\eta_{j}=\left\langle\eta, e_{j}\right\rangle, j=5,6$. We obtain that

$$
\begin{aligned}
\alpha_{g}(\partial, \partial)= & \frac{1}{\sqrt{\theta}}\left(\|\delta\|\left\langle A_{e_{3}} \partial, \partial\right\rangle-\omega(\partial)\left\langle\alpha(\partial, Z), e_{3}+i e_{4}\right\rangle\left(\eta_{5}-i \eta_{6}\right)\right)\left(\xi_{1}+i \xi_{2}\right) \\
& -\omega(\partial)\left\langle\alpha(\partial, Z), e_{3}+i e_{4}\right\rangle\left(e_{5}-i e_{6}\right) .
\end{aligned}
$$


On account of (12) and Lemma 3, we obtain

$$
\hat{\nabla}_{\partial}^{\perp} H_{g}=-\frac{2}{\theta^{3 / 2}} \partial \theta \xi_{1}+\frac{2}{\theta}\left(\tilde{\nabla}_{\partial}(Z-\delta)\right)^{N_{g} M} .
$$

Using (4) we find that

$$
\tilde{\nabla}_{\partial}(Z-\delta)=\partial+2 A_{\delta} \partial+2 \alpha(\partial, Z)+\nabla_{\partial}^{\perp} \eta
$$

It follows that

$$
\left\langle\tilde{\nabla}_{\partial}(Z-\delta), \xi_{1}\right\rangle=\frac{1}{\sqrt{\theta}}\left(\langle\partial, Z\rangle+\left\langle\nabla_{\partial}^{\perp} \delta, \eta\right\rangle\right)
$$

and

$$
\left\langle\tilde{\nabla}_{\partial}(Z-\delta), \xi_{2}\right\rangle=-\frac{1}{\sqrt{\theta}}\left(i\langle\partial, Z\rangle+\left\langle\nabla_{\partial}^{\perp} \mathcal{J} \delta, \eta\right\rangle\right) .
$$

Using (4) we obtain that

$$
\partial \theta=2\langle\partial, Z\rangle+2\left\langle\nabla_{\partial}^{\perp} \delta, \eta\right\rangle
$$

and from (7) that

$$
\left(\nabla_{\partial}^{\perp} \eta\right)^{\left(N_{1}^{f}\right)^{\perp}}=\left(\nabla_{\partial}^{\perp} g\right)^{\left(N_{1}^{f}\right)^{\perp}}-\left(\nabla_{\partial}^{\perp} \delta\right)^{\left(N_{1}^{f}\right)^{\perp}}=-\|\delta\| \omega(\partial)\left(e_{5}-i e_{6}\right) .
$$

It follows easily that

$$
\hat{\nabla}_{\partial}^{\perp} H_{g}=-\frac{2}{\theta^{3 / 2}}\left(\left(\langle\partial, Z\rangle+\|\delta\| \omega(\partial)\left(\eta_{5}-i \eta_{6}\right)\right)\right)\left(\xi_{1}+i \xi_{2}\right)-\frac{2}{\theta}\|\delta\| \omega(\partial)\left(e_{5}-i e_{6}\right) .
$$

Hence, we conclude from (15) and (16) that $g$ is S-Willmore if and only if

$$
\omega(\partial)\left(\|\delta\|^{2}\left\langle A_{e_{3}} \partial, \partial\right\rangle+\langle\partial, Z\rangle\left\langle\alpha(\partial, Z), e_{3}+i e_{4}\right\rangle\right)=0 .
$$

But $\omega(\partial) \neq 0$ since, otherwise, we have from (77) that $N_{1}^{f}$ is parallel in the normal bundle, which has been excluded. It follows easily that $g$ is S-Willmore if and only if $\kappa \theta=0$, but this is not possible.

\section{Final comments}

Changing the origin with respect to which the pedal of $f: M^{2} \rightarrow \mathbb{R}^{n}$ is taken amounts to add a constant vector to $f$. Given $c \in \mathbb{R}$ and $v \in \mathbb{R}^{n}$, the pedal surface $g_{c, v}=(c f+v)^{\perp}$ of $c f+v$ is a superconformal surface which is conformal to $f$. Decomposing $f$ and $v$ into its tangent and normal components

$$
f=Z+g \text { and } v=V+v^{\perp}
$$


we have that $g_{c, v}=c g+v^{\perp}$. When $f$ is 2-isotropic, it turns out that $g_{0, v}=v^{\perp}$ is superconformal which can be proved by a similar computations as the one given for $g$. But in this case, the image of $g_{0, v}=v^{\perp}$ by an inversion centered at $v$ is a minimal surface, hence 1-isotropic.

The superconformal surface $g$ constructed in Theorem 1 not only satisfies part $(i i)$ but a much stronger condition, namely, for any surface conformally equivalent to $g$ the rank of its first normal bundle is three. We sketch a proof of this fact but only in the special case of the pedal surface to a substantial 3-isotropic surface $f$ in $\mathbb{R}^{n}$ with $n \geq 8$.

Consider the immersion $\tilde{g}=\mathcal{I} \circ g$ where $\mathcal{I}$ is the inversion with respect to a sphere of radius $R$ centered at $p_{0}$. Then,

$$
\alpha_{\tilde{g}}(X, Y)=\frac{1}{R^{2}} \mathcal{P}\left(\left\|g-p_{0}\right\|^{2} \alpha_{g}+2\langle X, Y\rangle\left(g-p_{0}\right)^{\perp}\right)
$$

where $\mathcal{P}: N_{g} M \rightarrow N_{\tilde{g}} M$ is the vector bundle isometry already discussed. Then,

$$
N_{1}^{\tilde{g}}=\operatorname{span}\left\{\mathcal{P} \mu_{1}, \mathcal{P} \mu_{2}, \mathcal{P} \mu_{3}\right\}
$$

where

$$
\begin{aligned}
& \mu_{1}=\frac{1}{\sqrt{\theta}}\left(\left(\|\delta\|-\omega(Z) \eta_{5}-\omega(J Z) \eta_{6}\right) \xi_{1}+\left(\omega(J Z) \eta_{5}-\omega(Z) \eta_{6}\right) \xi_{2}\right)-\omega(Z) e_{5}-\omega(J Z) e_{6}, \\
& \mu_{2}=\frac{1}{\sqrt{\theta}}\left(\left(\omega(Z) \eta_{6}-\omega(J Z) \eta_{5}\right) \xi_{1}+\left(\|\delta\|-\omega(Z) \eta_{5}-\omega(J Z) \eta_{6}\right) \xi_{2}\right)-\omega(J Z) e_{5}+\omega(Z) e_{6}, \\
& \mu_{3}=\frac{1}{\sqrt{\theta}}\left(\left(\left\|g-p_{0}\right\|^{2}-\|\delta\|^{2}-\left\langle p_{0}, Z-\delta\right\rangle\right) \xi_{1}-\left\langle p_{0}, J Z+\mathcal{J} \delta\right\rangle \xi_{2}\right)+\eta-\left(p_{0}\right)^{\left(N_{1}^{f}\right)^{\perp}} .
\end{aligned}
$$

Suppose that $\lambda_{1} \mu_{1}+\lambda_{2} \mu_{2}+\lambda_{3} \mu_{3}=0$ with $\left(\lambda_{1}, \lambda_{2}, \lambda_{3}\right) \neq(0,0,0)$. Then,

$$
\lambda_{3}\left(\eta-p_{0}\right)^{\left(N_{1}^{f} \oplus N_{2}^{f}\right)^{\perp}}=0 .
$$

If $\lambda_{3}=0$ then $\omega=0$, a contradiction. Thus $\lambda_{3} \neq 0$ and hence $\eta-p_{0} \in T M \oplus N_{1}^{f} \oplus N_{2}^{f}$. We obtain that

$$
\alpha(X, W)=-\nabla_{X}^{\perp} \xi-\nabla_{X}^{\perp} \zeta
$$

where $p_{0}=W+\xi+\zeta, W \in T M, \xi \in N_{1}^{f}$ and $\zeta \in\left(N_{1}^{f}\right)^{\perp}$.

We claim that $\eta=\zeta$. Set $\eta=\zeta+\nu$ where $\nu \in N_{2}^{f}$. The above combined with (4) yield $\nabla_{X}^{\perp}(\xi-\delta-\nu) \in N_{1}^{f}$ and, consequently, $\nabla_{X}^{\perp} \nu \in N_{1}^{f} \oplus N_{2}^{f}$. Since $f$ is 3-isotropic, we have $\nabla_{X}^{\perp} \mathcal{J} \nu=\mathcal{J}\left(\nabla_{X}^{\perp} \nu\right)$. Thus, if $\nu \neq 0$, then $N_{1}^{f} \oplus N_{2}^{f}$ is parallel in the normal bundle, a contradiction. Hence $\eta=\zeta, \nabla_{X}^{\perp}(\xi-\delta) \in N_{1}^{f}$ and $\nabla_{X}^{\perp} \mathcal{J}(\xi-\delta) \in N_{1}^{f}$. Since $f$ is substantial, we obtain that $\xi=\delta$ and, consequently, that $\alpha(X, W-Z)=0$. But this gives that $W=Z$, a contradiction. 


\section{References}

[1] J. Barbosa, On minimal immersions of $S^{2}$ into $S^{2 m}$. Trans. Amer. Math. Soc. 210 (1975), 75-106.

[2] J. Bolton, F. Pedit and L. Woodward, Minimal surfaces and the affine Toda field model. J. Reine Angew. Math. 459 (1995), 119-150.

[3] R. Bryant, Submanifolds and special structures on the octonians. J. Differential Geom. 17 (1982), 185-232.

[4] E. Calabi, Minimal immersions of surfaces in Euclidean spheres. J. Differential Geom. 1 (1967), 111-125.

[5] C. C. Chen, The generalized curvature ellipses and minimal surfaces. Bull. Acad. Sinica 11 (1983), 329-336.

[6] S. S. Chern, On the minimal immersions of the two-sphere in a space of constant curvature. Problems in Analysis, 27-40. Princeton: University Press 1970.

[7] M. Dajczer and L. Florit, A Class of austere submanifolds. Illinois Math. J. 45 (2001), 735-755.

[8] M. Dajczer and D. Gromoll, The Weierstrass representation for complete minimal real Kaehler submanifolds. Invent. Math. 119 (1995), 235-242.

[9] M. Dajczer and R. Tojeiro, All superconformal surfaces in $\mathbb{R}^{4}$ in terms of minimal surfaces. Math. Z. 261 (2009), 869-890.

[10] M. Dajczer and Th. Vlachos, The dual superconformal surface. See http://arxiv.org/abs/1401.1291

[11] N. Ejiri, Willmore surfaces with a duality in $\mathbb{S}^{n}(1)$. Proc. London Math. Soc. 57 (1988), 383-416.

[12] "Enzyklopadie der mathematischen Wissenschaften mit ihrer Anwendungen" III D 2.1 Teubner. Leipzig, 1950.

[13] I. Guadalupe and L. Rodríguez, Normal curvature of surfaces in space forms. Pacific J. Math. 106 (1983), 95-103.

[14] E. Hulett. Harmonic superconformal maps of surfaces in $H^{n}$. J. Geom. Phys. 42 (2002), 139-165.

[15] R. Miyaoka, The family of isometric superconformal harmonic maps and the affine Toda equations. J. Reine Angew. Math. 481 (1996), 1-25. 
[16] C. Moore and E. Wilson, A general theory of surfaces. J. Nat. Acad Proc. 2 (1916), 273-278.

[17] C. Moore and E. Wilson, Differential geometry of two-dimensional surfaces in hyperspaces. Proc. of the Academy of Arts and Sciences, 52 (1916), 267-368.

[18] B. Rouxel, Some geometrical properties of superconformal and superminimal surfaces in $\mathbb{E}^{4}$. Proceedings of the Conference RIGA 2011 Riemannian Geometry and Applications Bucharest, Romania (2011), 255-260.

[19] G. Solmon "A treatise on the analytic geometry of three dimensions". New York, Chelsea, 1958-65.

[20] M. Spivak, "A Comprehensive Introduction to Differential Geometry". Vol. IV. Berkeley: Publish or Perish, 1979.

[21] Th. Vlachos, Minimal surfaces, Hopf differentials and the Ricci condition. Manuscripta Math. 126 (2008), 201-230.

[22] P. Wintgen, Sur l'inegalité de Chen-Willmore. C. R. Acad. Sci. Paris T. Ser. A 288 (1979), 993-995.

IMPA - Estrada Dona Castorina, 110 22460-320 - Rio de Janeiro - Brazil

E-mail: marcos@impa.br
Univ. of Ioannina - Math. Dept. 45110 Ioannina - Greece

E-mail: tvlachos@uoi.gr 